Cite this: J. Mater. Chem. A, 2013, 1, 11887

Received 13th June 2013

Accepted 31st July 2013

DOI: $10.1039 / c 3 t a 12302 b$

www.rsc.org/MaterialsA

\section{Low fractions of ionic liquid or poly(ionic liquid) can activate polysaccharide biomass into shaped, flexible and fire-retardant porous carbons $\dagger$}

\author{
Yongjun Men, ${ }^{\mathrm{a}}$ Miriam Siebenbürger, ${ }^{\mathrm{b}}$ Xunlin Qiu, ${ }^{\mathrm{c}}$ Markus Antonietti ${ }^{\mathrm{a}}$ \\ and Jiayin Yuan*a
}

Sugar-based molecules and polysaccharide biomass can be turned into porous functional carbonaceous products at comparably low temperatures of $400{ }^{\circ} \mathrm{C}$ under a nitrogen atmosphere in the presence of an ionic liquid (IL) or a poly(ionic liquid) (PIL). The IL and PIL act as "activation agents" with own structural contribution, and effectively promote the conversion and pore generation in the biomaterials even at a rather low doping ratio (7 wt\%). In addition, this "induced carbonization" and pore forming phenomenon enables the preservation of the biotemplate shape to the highest extent and was employed to fabricate shaped porous carbonaceous materials from carbohydrate-based biotemplates, exemplified here with cellulose filter membranes, coffee filter paper and natural cotton. These carbonized hybrids exhibit comparably good mechanical properties, such as bendability of membranes or shape recovery of foams. Moreover, the nitrogen atoms incorporated in the final products from the IL/PIL precursors further improve the oxidation stability in the fire-retardant tests.

\section{Introduction}

Bio-based materials derived from plants and lower organisms are an important class of renewable and abundant resources that regain rapidly increasing interest in both the scientific community and industrial production, due to sustainability issues. ${ }^{1-6}$ The capability of making functional materials entirely or partially from biomass is a part of humankind's ancient history and is how technology started. Excitingly, this concept is returning into the focus of modern material design and has already made a prominent impact on current research..$^{7-14}$ In particular, most natural materials are able to provide unique and sophisticated hierarchical structures and morphologies that are by far superior to most man-made constructions. Such refined structures can be immediately adopted as biotemplates or construction units to fabricate products with superior performance, as recently illustrated by Mann and other groups. ${ }^{15-18}$

Activated carbon is a class of widely used absorbents in a multitude of environmental applications for wastewater

${ }^{a}$ Department of Colloid Chemistry, Max-Planck-Institute of Colloids and Interfaces, D-14476 Potsdam, Germany. E-mail: jiayin.yuan@mpikg.mpg.de; Fax: +49-3315679502; Tel: +49-331-5679552

${ }^{b}$ Soft Matter and Functional Materials, Helmholtz-Zentrum Berlin für Materialien und Energie, D-14109 Berlin, Germany

'Department of Physics and Astronomy, University of Potsdam, D-14476 Potsdam, Germany

$\dagger$ Electronic supplementary information (ESI) available: Videos of the dye sorption experiment and the fire-retardant experiment. See DOI: 10.1039/c3ta12302b treatment, gas separation and purification, and more. ${ }^{19}$ It features large surface area, good chemical stability, oxygen-rich surface functionalities, and open pore structures ideal for the adsorption of large molecules. Typically, activated carbon can be produced from carbon-rich precursors, such as coal, lignite and agricultural waste via physical and chemical activation..$^{20,21}$ The former method deals with the pyrolysis of the precursors and gasification of the resulting char in steam or carbon dioxide. ${ }^{22}$ In the latter method, carbon precursors are impregnated with excessive chemical reagents, such as phosphoric acid, zinc chloride, and alkaline hydroxides, and pyrolysed under an inert gas atmosphere at temperatures often between 600 and $800{ }^{\circ} \mathrm{C} .{ }^{23}$ In addition, a purification process is required to remove the residue activation chemicals. It is therefore highly desired to develop new activation agents, which work at low doping ratios and low temperatures, and can ideally be incorporated into the final carbon products to avoid the postsynthesis purification.

In this article, we report on the facile preparation of porous carbonaceous architectures with well-defined shape and complex structure by lowering the activation temperature of bio(macro)molecules and plant biostructures down to the point of structural preservation with the assistance of imidazoliumbased ionic liquids (ILs) or poly(ionic liquid)s (PILs). Surprisingly it was found that even at rather low added amounts, ILs or PILs can efficiently promote the carbonization and micropore generation in the bio-entities without destroying the structural skeleton pre-existing in the biotemplates down to very fine structural details. Finally following the biomass-activation 
procedure the ILs or PILs favorably ended up as porous nitrogen-doped carbon in the final porous product, i.e. no need to remove any residue activation agent, and it additionally improved the fire-retardancy.

\section{Experimental section}

\section{Materials}

1-Vinylimidazole (99\%), bromoacetonitrile (97\%), 1,3-bis(cyanomethyl)imidazolium bis(trifluoromethylsulfonyl)imide ( $\geq 94 \%$ ), lithium bis(trifluoromethanesulfonyl)imide (99.95\%), $\alpha$ - and $\beta$-cyclodextrin ( $\geq 97 \%)$, D- $(+)$-glucose (99.5\%), starch (puriss. p.a., from potato), cellulose (fibers, particle size: medium), poly(ethylene oxide) $\left(M_{\mathrm{v}} \sim 600000 \mathrm{~g} \mathrm{~mol}^{-1}\right)$, poly(vinyl alcohol) (Mw $\sim 89000-98000 \mathrm{~g} \mathrm{~mol}^{-1}$ ), polystyrene $\left(\mathrm{Mw} \sim 192000 \mathrm{~g} \mathrm{~mol}^{-1}\right)$ and poly(acrylic acid) $\left(M_{\mathrm{v}} \sim 450000 \mathrm{~g} \mathrm{~mol}^{-1}\right)$, crystal violet (dye content $\geq 90 \%$ ), and methylene blue were obtained from SigmaAldrich and used without further purification. 2,2'-Azobis(2-methylpropionitrile) (98\%, Aldrich) was recrystallized from methanol. An ionic liquid monomer, 3-cyanomethyl-1-vinyl imidazolium bromide (CMVImBr) was prepared via quaternization of 1-vinylimidazole with bromoacetonitrile, according to our previous paper. ${ }^{24}$ The solvents and other materials were used as received.

\section{Polymerization of the CMVImBr monomer}

Monomer 3-cyanomethyl-1-vinyl imidazolium bromide (16.2 g) and AIBN (320 mg) were dissolved in $160 \mathrm{~mL}$ of DMSO in a $500 \mathrm{~mL}$ Schlenk flask equipped with a magnetic stirring bar. After complete dissolution of the monomer, the solution was sealed, degassed by vacuum and washed with argon several times. The polymerization was conducted in argon at $90^{\circ} \mathrm{C}$ under magnetic stirring for 24 hours. After cooling down, the reaction mixture was purified by precipitation twice into acetone. A yellowish powder was obtained and dried at $60^{\circ} \mathrm{C}$ under vacuum to constant weight. Yield: $80 \% .{ }^{1} \mathrm{H}-\mathrm{NMR}$ (DMSO-d6, $\delta$, ppm): 8.9$9.6(1 \mathrm{H}), 7.0-8.4(2 \mathrm{H}), 5.2-5.7(2 \mathrm{H}), 3.5-4.6(1 \mathrm{H}), 1.9-2.7(2 \mathrm{H})$.

\section{Anion exchange of the PCMVImTf ${ }_{2} \mathbf{N}$ polymer}

$6 \mathrm{~g}$ of poly(3-cyanomethyl-1-vinyl imidazolium bromide) was added to $800 \mathrm{~mL}$ of water under gentle heating. After complete dissolution and cooling down, $200 \mathrm{~mL}$ of an aqueous solution containing 1.05 molar equivalents of lithium bis(trifluoromethanesulfonyl)imide salt was added under vigorous stirring. The solution became turbid and precipitation occurred. The precipitate was filtered, washed with pure water several times and dried at $60^{\circ} \mathrm{C}$ to constant weight under high vacuum $\left(1 \times 10^{-2}\right.$ mbar $)$. Yield: $70 \%$.

\section{Loading of an IL/PIL onto various molecular substrates}

To load an IL/PIL onto $\beta$-CD, a mixture of IL or PIL ( $0.50 \mathrm{~g})$ and different amounts of $\beta$-CD powder at designed weight ratios (entries 3-8, 17 and 18 in Table 1) was finely ground and mixed in an alumina crucible. The same procedure was applied also to glucose, starch, cellulose, and synthetic polymers (PAA, PEG,
Table 1 Characterization of the carbonization products of biomaterials and ionic liquid or poly(ionic liquid) in defined ratios at $400{ }^{\circ} \mathrm{C}$ under a nitrogen atmosphere (heating rate: $5^{\circ} \mathrm{C} \mathrm{min}^{-1}$ )

\begin{tabular}{|c|c|c|c|c|c|c|}
\hline Entry & Component & $\begin{array}{l}\text { IL or PIL } \\
\text { fraction } \\
(w t \%)\end{array}$ & $\begin{array}{l}S_{\mathrm{BET}} \\
\left(\mathrm{m}^{2} \mathrm{~g}^{-1}\right)\end{array}$ & $\begin{array}{l}\text { Yield } \\
(\%)\end{array}$ & $\begin{array}{l}\mathrm{N} \\
(\%)\end{array}$ & $\begin{array}{l}\mathrm{H} \\
(\%)\end{array}$ \\
\hline 1 & IL & 0 & 570 & 39.1 & 31.5 & 2.5 \\
\hline 2 & $\beta-C D$ & 100 & 0 & 26.0 & 0.4 & 4.0 \\
\hline 3 & $\mathrm{IL}+\beta-\mathrm{CD}$ & 21 & 724 & 38.4 & 22.9 & 2.5 \\
\hline 4 & $\mathrm{IL}+\alpha-\mathrm{CD}$ & 21 & 735 & 35.9 & 23.0 & 2.3 \\
\hline 5 & $\mathrm{IL}+\beta-\mathrm{CD}$ & 57 & 503 & 39.8 & 12.9 & 2.9 \\
\hline 6 & $\mathrm{IL}+\beta-\mathrm{CD}$ & 73 & 486 & 38.9 & 9.3 & 2.3 \\
\hline 7 & $\mathrm{IL}+\beta-\mathrm{CD}$ & 84 & 266 & 37.6 & 5.4 & 3.3 \\
\hline 8 & $\mathrm{IL}+\beta-\mathrm{CD}$ & 93 & 116 & 35.8 & 3.0 & 3.6 \\
\hline 9 & IL + glucose & 57 & 586 & 36.9 & 15.9 & 2.1 \\
\hline 10 & $\mathrm{IL}+$ starch & 57 & 605 & 37.6 & 12.9 & 2.7 \\
\hline 11 & IL + cellulose & 57 & 623 & 35.9 & 13.3 & 2.6 \\
\hline 12 & $\mathrm{IL}+\mathrm{PEG}$ & 57 & 22 & 22.8 & 14.4 & 2.7 \\
\hline 13 & $\mathrm{IL}+\mathrm{PAA}$ & 57 & 40 & 25.6 & 10.3 & 2.7 \\
\hline 14 & $\mathrm{IL}+\mathrm{PS}$ & 57 & 57 & 19.7 & 23.8 & 2.0 \\
\hline 15 & $\begin{array}{l}\text { IL + filter } \\
\text { membrane }\end{array}$ & 57 & 680 & 36.9 & 13.4 & 3.0 \\
\hline 16 & $\begin{array}{l}\mathrm{IL}+\text { coffee } \\
\text { paper }\end{array}$ & 57 & 720 & 37.5 & 15.5 & 3.3 \\
\hline 17 & $\mathrm{PIL}+\beta-\mathrm{CD}$ & 57 & 530 & 32.2 & 9.3 & 2.9 \\
\hline 18 & $\mathrm{PIL}+\beta-\mathrm{CD}\left(500^{\circ} \mathrm{C}\right)$ & 73 & 534 & 31.5 & 7.2 & 2.7 \\
\hline 19 & $\begin{array}{l}\text { PIL + filter } \\
\text { membrane }\end{array}$ & 88 & 400 & 35.6 & 2.1 & 2.7 \\
\hline 20 & PIL + cotton & 88 & 350 & 35.4 & 2.2 & 2.9 \\
\hline
\end{tabular}

and PS), in which the IL occupied $43 \mathrm{wt} \%$ in the mixture (entries 9-14 in Table 1).

\section{Loading of an IL/PIL onto the filter membrane and coffee paper}

To load an IL onto the biotemplates (entries 15 and 16), an IL $(1 \mathrm{~g})$ solution in ethanol $(10 \mathrm{~mL})$ was spread onto a cut filter membrane or coffee filter paper $(2.33 \mathrm{~g})$, which was dried at $80{ }^{\circ} \mathrm{C}$.

To load a PIL onto the filter membrane (entry 19), a dried filter membrane $(3 \mathrm{~g})$ was immersed in the PIL solution in acetonitrile ( $10 \mathrm{wt} \%$, viscosity $=1.4 \mathrm{mPa} \mathrm{s}$ ). After 5 minutes, it was taken out. The residue solution on the surface was wiped off and the rest was removed by vacuum filtration. The filter membrane was then dried at $80{ }^{\circ} \mathrm{C}$ until constant weight. The fraction of the PIL was calculated as the weight difference between the filter membrane and the PIL-loaded filter membrane.

\section{Loading of a PIL onto cotton}

$3 \mathrm{~g}$ of dried cotton was immersed in a PIL solution in acetonitrile $(10 \mathrm{wt} \%)$ for $5 \mathrm{~min}$. The wet cotton was then pressed into a round disc membrane in a Buchner funnel. The residue solution was removed by vacuum filtration via squeezing the wet cotton tightly into a dense foam. The cotton was then dried at $80{ }^{\circ} \mathrm{C}$ until constant weight. The fraction of the PIL was 
calculated as the weight difference between the cotton and the PIL-loaded cotton.

\section{Carbonization process}

For carbonization, an IL, a PIL, or their mixtures with biomaterials were loaded into an alumina crucible in an alumina tube furnace, and carbonized under nitrogen flow. The sample was heated up to $400{ }^{\circ} \mathrm{C}\left(5{ }^{\circ} \mathrm{C} \min ^{-1}\right)$ and kept for $1 \mathrm{~h}$ at $400{ }^{\circ} \mathrm{C}$. In the case of entry 18 , the sample was heated to $500{ }^{\circ} \mathrm{C}\left(5^{\circ} \mathrm{C}\right.$ $\min ^{-1}$ ) and kept for $1 \mathrm{~h}$ at $500{ }^{\circ} \mathrm{C}$. Afterwards, the furnace was slowly cooled down to room temperature.

\section{Dye sorption and fire-retardant experiments}

For the dye-removal experiment, carbonaceous foam derived from the PIL/cotton composite was cut into a designed shape $(0.8 \mathrm{~cm} \times 0.8 \mathrm{~cm} \times 0.2 \mathrm{~cm})$, and immersed in a methylene blue aqueous solution $\left(10 \mathrm{mg} \mathrm{L}^{-1}\right)$. After a few seconds, the foam was taken out, and squeezed to repel the clean solution out of the foam. For removing crystal violet from aqueous solution, $20 \mathrm{mg}$ of the carbonaceous cotton foam or PIL/cotton composite foam was added to crystal violet solution $\left(10 \mathrm{mg} \mathrm{L}^{-1}\right)$, and the mixture was shaken for $5 \mathrm{~min}$. For the fire-retardant experiment, cylindrical carbonaceous foams were obtained directly by carbonization of cotton or PIL-coped cotton within a quasi-tube.

\section{Characterization methods}

Nitrogen sorption experiments were performed with a Quantachrome Autosorb-1 or Quadrasorb at liquid nitrogen temperature, and data analysis was performed with Quantachrome software. The surface area was calculated using the BET equation. The pore size distribution was calculated using the Barrett-Joyner-Halenda (BJH) method. The samples were degassed at $120{ }^{\circ} \mathrm{C}$ for $15 \mathrm{~h}$ before measurements.

Elemental analysis was performed for carbon, hydrogen, sulphur and nitrogen using a Vario EL Elementar. TGA experiments were accomplished on a Netzsch TG209-F1 apparatus at a heating rate of $10 \mathrm{~K} \mathrm{~min}^{-1}$ under nitrogen. SEM images were obtained on a LEO 1550-Gemini instrument after sputtering with platinum.

Mechanical tensile testing was performed at room temperature using a Zwick Z005 (Zwick GmbH \& Co KG, Germany) tensile testing machine equipped with a $10 \mathrm{~N}$ load cell. All samples had identical geometry, the dog bone shape, achieved using a cutting die of a standard shape (DIN 53504) for the pure tensile uniaxial strain experiment. The viscosity measurement was performed with a MCR 301 rheometer (Anton Paar) attached with a coaxial cylindrical double-gap geometry (inner cup radius of $11.910 \mathrm{~mm}$, outer cup radius of $13.795 \mathrm{~mm}$, inner bob radius of $12.330 \mathrm{~mm}$, outer bob radius of $13.334 \mathrm{~mm}$ and an internal bob length of $42 \mathrm{~mm}$ ) at $25^{\circ} \mathrm{C}$.

\section{Results and discussion}

ILs are substances composed of paired, mostly organic cations and anions which melt below $100{ }^{\circ} \mathrm{C} .{ }^{24-26}$ PILs are ionic polymers obtained by polymerization of monomeric ILs. ${ }^{27-29}$ The<smiles>N#CCn1cc[n+](CC#N)c1</smiles><smiles>CC(C)(C)CC(n1cc[n+](CC#N)c1)C(C)(C)C</smiles><smiles>O=S(=O)(NS(=O)(=O)C(F)(F)F)C(F)(F)F</smiles><smiles>O=S(=O)(NS(=O)(=O)C(F)(F)F)C(F)(F)F</smiles>

Scheme 1 Chemical structures of the ionic liquid and poly(ionic liquid) applied as chemical activation agents.

chemical structures of the IL and PIL studied in detail here are illustrated in Scheme 1. The unique structural feature of both substances is that either one or two cyanomethyl-groups are attached directly to the imidazolium cation ring, while a largesized anion, bis(trifluoromethylsulfonyl)imide, $\mathrm{Tf}_{2} \mathrm{~N}$, was chosen for charge neutralization. This unique chemical combination is known to readily form microporous carbons upon pyrolysis of pure IL or PIL under nitrogen at unexpectedly low temperatures even without any external template, as reported by Dai et al. ${ }^{30-34}$ In our study, when the IL (3-cyanomethyl-1-vinylimidazolium $\mathrm{Tf}_{2} \mathrm{~N}$ ) was heated to only $400{ }^{\circ} \mathrm{C}$ at a heating rate of $5{ }^{\circ} \mathrm{C} \min ^{-1}$ under a nitrogen atmosphere, it produced a carbonaceous framework (entry 1 in Table 1) with an oven yield of $39.1 \mathrm{wt} \%$. The $\mathrm{N}_{2}$ sorption measurement (Fig. 1) revealed that the product is essentially microporous with a specific surface area $\left(S_{\mathrm{BET}}\right) \sim 570 \mathrm{~m}^{2} \mathrm{~g}^{-1}$, which is in good accordance with the earlier reported values. ${ }^{32}$ Here, the ultramicropores $(<2 \mathrm{~nm})$ were left by the thermal removal of the large $\mathrm{Tf}_{2} \mathrm{~N}$ anion, i.e. a molecular templating mechanism, also explaining the weight loss. This is supported by the observation of a low sulfur content in these products.

$\beta$-Cyclodextrin, $\beta$-CD, here used as a cyclic oligosaccharide, on the other hand generates only a compact and nonporous solid when processed in the same manner. Unexpectedly, when $\beta$-CD was mixed in $21 \mathrm{wt} \%$ with this IL (molar ratio of

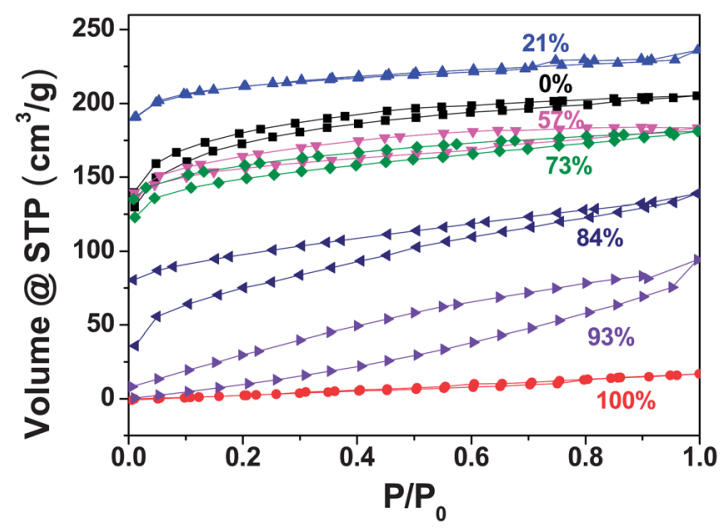

Fig. 1 Nitrogen sorption isotherms of carbonaceous products (entries 1-3 and $5-8$ in Table 1) obtained at $400{ }^{\circ} \mathrm{C}$ from a mixture of IL with different weight fractions of $\beta-C D$. 
$\beta$-CD/IL $=1: 10$ ), a microporous network formed with an even higher $S_{\mathrm{BET}} \sim 724 \mathrm{~m}^{2} \mathrm{~g}^{-1}$, while the oven yield remained practically untouched (entry 3 in Table 1). In a word, the mixing of $\beta$-CD and IL seems to promote pore formation. Only the nitrogen content drops from 39.1 to $22.9 \mathrm{wt} \%$ when $\beta$-CD was added. This is understandable, as $\beta-\mathrm{CD}$ is a nitrogen-free compound. The same result was obtained for $\alpha$-CD as well (entry 4 in Table 1).

The $\beta$-CD-content was systematically increased in the carbonization mixture. At a $\beta$-CD weight fraction of $57 \mathrm{wt} \%$, yet a microporous product with $S_{\mathrm{BET}} \sim 503 \mathrm{~m}^{2} \mathrm{~g}^{-1}$ (entry 5 in Table 1) was formed, which is very close to that of the pure IL. Upon replacing more IL with $\beta$-CD in the mixture, the $S_{\mathrm{BET}}$ values of the formed products drop accordingly. Nevertheless, even at the highest $\beta$-CD weight content of $93 \mathrm{wt} \%$ in the mixture, the pyrolyzed product remained porous $\left(S_{\mathrm{BET}} \sim 116 \mathrm{~m}^{2}\right.$ $\mathrm{g}^{-1}$, entry 8 in Table 1 ), although with a non-homogeneous pore distribution. Obviously, $\beta-C D$ turns into (micro)porous networks with the assistance of an IL, and the IL acts as a "poreinducing agent" at $400{ }^{\circ} \mathrm{C}$. The oven yield remains almost constant throughout all experiments, ending up with $35.8 \mathrm{wt} \%$ at a high $\beta$-CD content of $93 \mathrm{wt} \%$ in the starting mixture.

To create a more complete picture of this unique behaviour, a wider reaction temperature range from 300 to $1000{ }^{\circ} \mathrm{C}$ was investigated at a constant $\beta$-CD content of $73 \mathrm{wt} \%$. As shown in Fig. 2, the highest $S_{\mathrm{BET}}$ value was observed at $500{ }^{\circ} \mathrm{C}$. The solid achieved below $400{ }^{\circ} \mathrm{C}$ was nonporous, and above $800{ }^{\circ} \mathrm{C}$ it seems that the pores formed earlier at intermediate temperatures collapse. The effect of the dwelling time was also studied at $400{ }^{\circ} \mathrm{C}$. The microporous framework, once built up, was
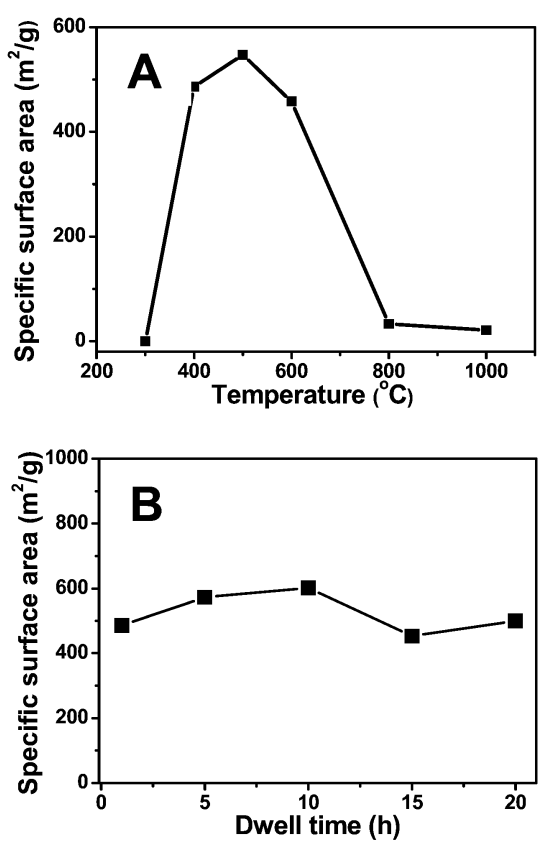

Fig. 2 (A) Plot of the specific surface area vs. the carbonization temperature. (B) Plot of specific surface area vs. the dwelling time. The sample was prepared by mixing $73 w t \%$ of $\beta$-CD with $27 w t \%$ of IL. Heating program: $5^{\circ} \mathrm{C} \mathrm{min}^{-1}$ to the final temperature and dwelling for different periods at the final temperature. proven to be stable upon further thermal annealing, and only a small deviation in $S_{\mathrm{BET}}$ was seen (Fig. 2).

To judge the generality, glucose, cellulose and starch were also employed as a carbohydrate base at a component fraction of $57 \mathrm{wt} \%$ (entries 9-11 in Table 1). As expected, all products presented a high surface area of around $600 \mathrm{~m}^{2} \mathrm{~g}^{-1}$ with a satisfactory oven yield exceeding $35 \%$. In contrast, when several synthetic polymers, such as poly(ethylene glycol) (PEG), polystyrene (PS) and poly(acrylic acid) (PAA), were treated in the same manner, the reaction ended up with poorly porous solids and low yields (entries 12-14 in Table 1).

As glucose and polysaccharides are abundant and industrially available for minimal prices as raw materials and "microstructured" templates (such as paper), respectively, the accelerated carbonization/activation function of ILs can be readily expanded to produce porous carbonaceous materials from a multiplicity of pre-shaped templates. For practical reasons, we complemented the IL with its polymer form, poly(3-cyanomethyl-1-vinyl-imidazolium $\left.\mathrm{Tf}_{2} \mathrm{~N}\right) \cdot{ }^{35-38}$ The advantage of PILs over ILs in this case lies in the superior processability of polymers, which allows us to coat also the inner surface of the hierarchical structures of biotemplates more conveniently, homogeneously and efficiently by simple impregnation; therefore a "dry" and touchable green body product is obtained for further processing. In contrast, it is difficult for ILs to form a thin film on a planar surface with thickness down to nanoscale, and dewetting is another problem due to their mobile/fluidic feature. The fact that a PIL also acts as an "activation agent" was quantified in a model reaction with a $57 \mathrm{wt} \% \beta-\mathrm{CD}$ mixture at $400{ }^{\circ} \mathrm{C}$. A microporous solid with $S_{\mathrm{BET}} \sim 530 \mathrm{~m}^{2} \mathrm{~g}^{-1}$ was received (entry 17 in Table 1 ). Similar results are obtained at $500{ }^{\circ} \mathrm{C}$ at $73 \mathrm{wt} \% \beta$-CD (entry 18 in Table 1 ).

The conversion of a biotemplate with extended shape was first tested with a commercially available cellulose filter membrane and coffee filter paper (entries 15, 16 and 19), both of which are made mainly of natural cellulose fibers. In the doping step, an IL solution in ethanol was spread onto the biotemplate. This was carried out with caution to avoid inhomogeneity of IL deposition. After $400{ }^{\circ} \mathrm{C}$ treatment, the $S_{\text {BET }}$ reached $680 \mathrm{~m}^{2} \mathrm{~g}^{-1}$ for the filter membrane and $720 \mathrm{~m}^{2} \mathrm{~g}^{-1}$ for the coffee filter. In the case of a PIL, the procedure is more convenient. The filter membrane or paper was directly immersed in a PIL solution to allow the PIL macromolecules to soak into the interstitial voids of the cellulose paper. After removal of the excess solution via soft vacuum filtration, the $\mathrm{PIL} /$ cellulose composite paper was dried at $80^{\circ} \mathrm{C}$ until constant weight to calculate the PIL content, which is $12 \mathrm{wt} \%$ (Fig. 3A). After thermal treatment at $400{ }^{\circ} \mathrm{C}$, a flat carbonaceous membrane with $S_{\text {BET }} \sim 400 \mathrm{~m}^{2} \mathrm{~g}^{-1}$ (Fig. 3B) was obtained (entry 19 in Table 1). The radial shrinkage of the membrane after pyrolysis was determined to be $c a$. $10-15 \%$. To our surprise, the membrane preserved essentially the mechanical flexibility of the original paper. As illustrated in Fig. 3C, it can be bent to a large extent at will and cut into different forms, such as stripes shown in Fig. 3D and E. This feature is of high convenience to assemble it into filtration and separation devices, which require high mechanical stability and flexibility. It is noteworthy that 

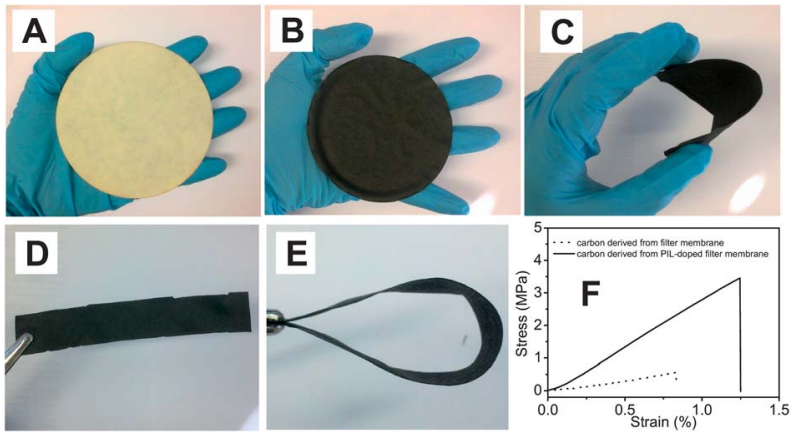

Fig. 3 Photographs of (A) PIL wetted filter paper, (B) after carbonization at $400{ }^{\circ} \mathrm{C}$, (C) bending test of the carbonaceous membrane paper, and (D) a carbonaceous stripe cut from a carbonaceous membrane. (E) The bending test of the stripe. (F) Plots of the tensile testing experiments of carbon membranes derived from the pure cellulose filter membrane (dotted line) and from the PILdoped filter membrane (solid line).

without IL/PIL doping, the carbonaceous membrane showed only little porosity and a rather unsatisfying mechanical behavior. Fig. $3 \mathrm{~F}$ shows the tensile testing experiment results. When produced from a PIL-doped filter membrane, the carbon membrane reaches not only a four times higher breaking strength but also a 50\% larger rupture strain. This clearly illustrates the onset of a new condensation chemistry.

Beside cellulose membranes or papers, natural cotton could also be taken to "carbonize" it under preservation of the outer shape and microtexture to end up in a condensed product with good mechanical performance (entry 20 in Table 1). The particular features of cotton are its very high absolute porosity and the assorted superior deformability, which can be pressed or engineered into various shapes (note that cotton is one of the oldest gas permeable sealant materials of chemistry). Typically, a handful of cotton in its original state (Fig. 4A) was soaked in a PIL solution for surface coating. The excess solution was mechanically pressed out or sucked away by vacuum filtration. After drying at room temperature, it was squeezed between two

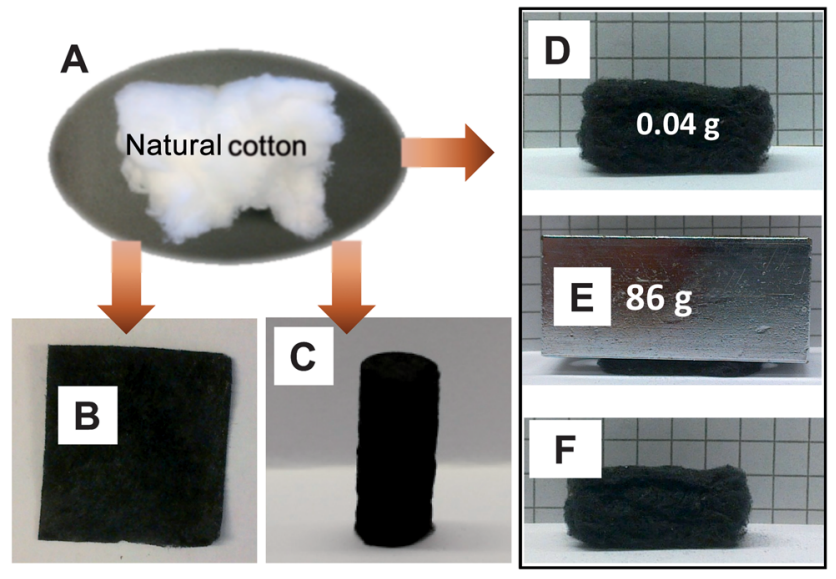

Fig. 4 Photographs of (A) natural cotton, (B) carbonaceous thin film derived from a PIL/cotton composite film, (C) carbonaceous monolith derived from a PIL/ cotton composite cylinder, and (D) cut carbonaceous cuboid from a carbonized $\mathrm{PIL} /$ cotton composite. ( $\mathrm{E}$ and $\mathrm{F}$ ) A shape retention test on the composite. glass plates or into a cavity and in situ heat-transferred into a film (Fig. 4B) or into a differently shaped monolithic object. A carbonaceous monolith (Fig. 4C) was for instance generated by densely pressing the coated cotton precursor into a test tube. Carbonizing the PIL/cotton composite in its original form produces an irregularly shaped fluffy foam that can be conveniently cut into any well-defined geometry, such as a cuboid geometry shown in Fig. 4D. The carbonaceous cuboid foam is again sponge-like and deformable. In a mechanical test shown in Fig. 4E and F, it recovers itself readily after being compressed by 2000 times of its own weight.

With the fairly good preservation of the macroscopic shape, it is an interesting question what occurs to the interior microscopic texture of the cellulose throughout pyrolysis. To analyze this, the intact filter paper, PIL-coated-filter paper and the carbonaceous membrane copy were subjected to scanning electron microscopy (SEM) characterization. Fibers of $10-40 \mu \mathrm{m}$ in diameter are clearly seen in these samples (Fig. 5A-C), essentially no changes of the microtexture are found even with careful observation. The fiber skeleton holds firmly its architecture when converted from cellulose to the carbonaceous fibers. Therefore, the described low temperature carbonization process retains the morphology well, both macro- and microscopically.

To illustrate one of the many possible functions of these biotemplate-derived porous carbonaceous materials, we performed dye sorption experiments in aqueous solutions (Fig. 6). The porous carbonaceous sponge derived from the cotton/PIL pad quickly absorbed the aqueous solution of methylene blue dye and clean water was released by squeezing it.

A video (Video 1) of the actual experiment is included in the ESI. $\dagger$ In an alternative example, an aqueous solution of methylene blue was decolorized after passing through a short column filled with the carbon sponge, which demonstrates the efficient removal of organic dyes from the aqueous phase. The PIL added to the cotton played an important role in accelerating the absorption kinetics. In a control experiment of absorbing crystal violet from aqueous solution, the porous carbon foam derived from cotton/PIL (Fig. 6F) presented a much faster kinetics in the dye sorption than the porous carbon foam derived from pure cotton (Fig. 6E).

Inertness against oxidation is another classical feature of $\mathrm{N}$-heterocycles and $\mathrm{N}$-doped carbons. ${ }^{39}$ Here, we tested our carbon foams (cylinders prepared at $400{ }^{\circ} \mathrm{C}$ from cotton with/ without PIL) by wetting the foam with $500 \mathrm{wt} \%$ of ethanol and

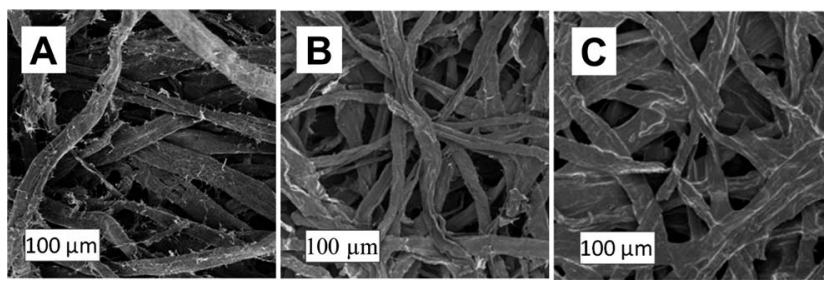

Fig. 5 SEM images of an intact cellulose filter membrane (A), a PIL-decorated cellulose filter membrane (B), and its corresponding carbonaceous membrane after PIL activation (C) 


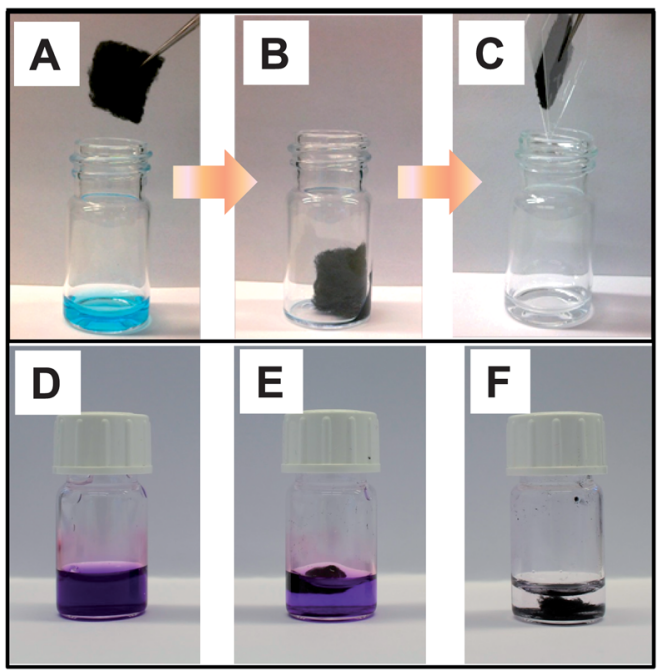

Fig. 6 (A-C) Photographs illustrating the sorption of methylene blue dye from aqueous solution by a carbonaceous foam derived from the PIL/cotton composite. (D-F) Photographs of the initial aqueous solution of crystal violet (D), 5 min after treatment by pure cotton-based carbon foam (E), and 5 min after treatment by a cotton/PIL-based carbon foam (F).

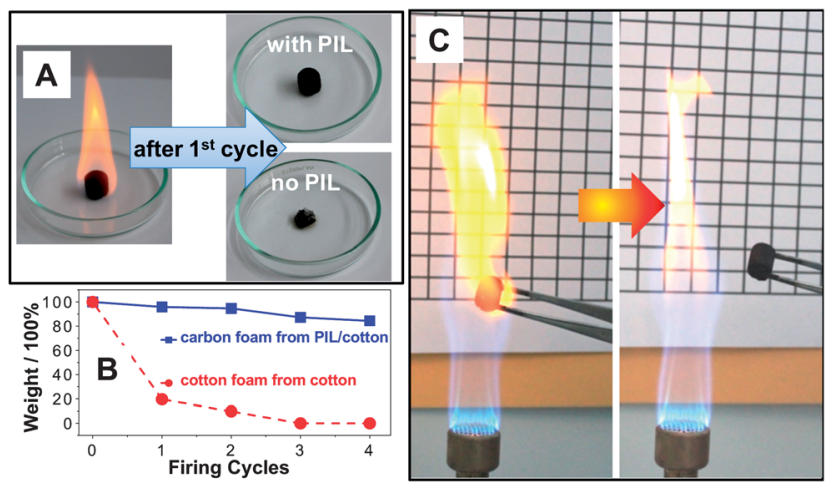

Fig. 7 (A) Photographs of the 1st firing cycle test of the carbon foams (absorb $500 \mathrm{wt} \%$ of ethanol and burn in air). Left: a burning ethanol-wetted foam. Right: foams after the 1st firing cycle test. (B) Plot of the carbon foam mass vs. the firing cycle. (C) Photographs illustrating the fire-retardancy of a carbonaceous foam by repeatedly firing the sample using a butane/propane gas burner.

burning it in air (Fig. 7A). This test was repeated 4 times to monitor the shape and mass change after each cycle. As observed, the carbon foam derived from pure cotton collapsed after the 1st cycle already, while the PIL/cotton carbon remained intact. The mass $v s$. firing cycle plot (Fig. 7B) indicates that the 1st firing cycle burnt away $80.3 \mathrm{wt} \%$ of the cotton-based carbon product, while only $4.1 \mathrm{wt} \%$ of mass loss occurred for the cotton/PIL carbon foam. Within 3 cycles, only the cotton/PIL carbon foam preserved its shape and $85 \mathrm{wt} \%$ of its mass. Under even harsh conditions, for examples, when repeatedly exposed to the flame of a butane/propane $(70: 30)$ gas burner, the foam maintained well its shape and size, without quenching fire (Fig. 7C). In a real-time video (Video 2 in the ESI $\dagger$ ), it is observed that the foam bottom could be turned to a red blaze in the central flame $\left(\sim 1300{ }^{\circ} \mathrm{C}\right)$, but quenched immediately when

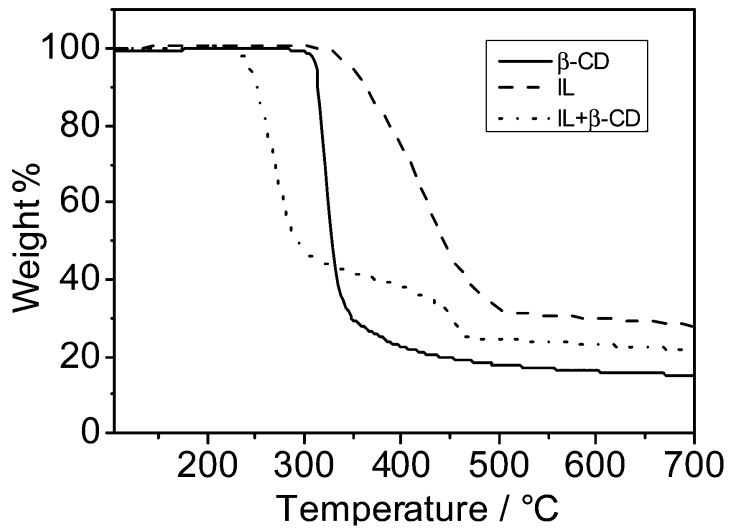

Fig. 8 TGA curves of IL, $\beta-C D$ and the mixture of IL/ $\beta-C D$ in a weight ratio of $43 /$ 57. The experiments were conducted under a nitrogen atmosphere at a heating rate of $10^{\circ} \mathrm{C} \mathrm{min}^{-1}$

pulled out (or even treated with blowing air). This illustrates that the material is even robust against co-firing, a really exceptional property for high surface area carbons.

The "low temperature carbonization/activation" function of ILs/PILs for biomolecules and biomass resembles a chemical activation process, but at much lower temperatures and lower chemical demand than standard carbonization. Certain intrinsic chemical reactions between the IL species and the sugar unit must occur at a molecular level at the described temperatures. Fig. 8 illustrates the thermogravimetric analysis (TGA) curves of IL, $\beta$-CD and a mixture of IL/ $\beta$-CD (weight ratio $\sim 43 / 57$, entry 5 in Table 1 ), which help understand the structural preservation of biotemplates during the carbonization. It is clearly found that the mixing lowers significantly the onset of mass loss, indicating that a new chemistry is involved. The primary weight loss starts at $240^{\circ} \mathrm{C}, c a .70^{\circ} \mathrm{C}$ lower than in $\beta$-CD and even $90^{\circ} \mathrm{C}$ than in the IL. This is exactly the critical and important temperature reduction as now carbonization sets in practically before the thermal degradation of cellulose starts, i.e. the biological structure can be preserved throughout the chemical conversions, while sintering is suppressed.

Dehydration of sugar with sulfuric acid is a well-known chemical reaction, and several groups reported earlier that sugar units could be catalytically dehydrated at reduced temperatures in some ILs. ${ }^{31,40}$ Our TGA analysis matches well with these findings, implying that the early weight loss of the IL/ $\beta$-CD mixture can be attributed to a catalytic dehydration step. Analysis of the fragmentation products indicated a rather complex decomposition scenario rather than simple water elimination. The nitrile groups of the IL moiety must play a key role in cross-linking the scaffold. ${ }^{30-33} \mathrm{~A}$ more detailed study on the ongoing chemical processes is in progress and will be reported elsewhere.

\section{Conclusions}

In conclusion, we demonstrated in this report a simple transformation of polysaccharide-based biomaterials into porous, shapable carbonaceous frameworks, simply by coating them with an IL/PIL, followed by thermal transformation under a 
nitrogen atmosphere at $400{ }^{\circ} \mathrm{C}$. The IL and PIL act as efficient carbonization/activation agents to promote conversion and pore generation in the biomaterial even at comparably low temperatures. Their role can be understood as an "activation agent" with own structural contribution. It was shown that through this route, useful carbonaceous products of different geometries could be obtained from similarly shaped biotemplates acting as "green bodies" for this process. To our surprise, the carbonized hybrid still offers comparably good mechanical properties, such as bendability of membranes or recovery of foams, which attributes some flexibility to the as-made product. Here, it must be stated that elasticity and microporosity usually exclude each other, structure-wise, both in polymers as well as in carbons. ${ }^{\mathbf{4 1}}$ The heteroatoms incorporated in the final products from the IL/ PIL precursors, such as nitrogen here, add extra value to the carbon products, as shown here by the brought in extraordinary oxidation stability. We believe that the concept of PIL activation opens an alternative avenue to produce appealing functional carbonaceous materials in a rather sustainable way.

\section{Acknowledgements}

The authors thank the Max Planck Society for the financial support. Y. Men would like to acknowledge the China Scholarship Council for a scholarship.

\section{Notes and references}

1 M.-M. Titirici and M. Antonietti, Chem. Soc. Rev., 2010, 39, 103-116.

2 Y. Zhai, Y. Dou, D. Zhao, P. F. Fulvio, R. T. Mayes and S. Dai, Adv. Mater., 2011, 23, 4828-4850.

3 L. Liu, D. He, G.-S. Wang and S.-H. Yu, Langmuir, 2011, 27, 7199-7206.

4 X.-L. Wu, T. Wen, H.-L. Guo, S. Yang, X. Wang and A.-W. Xu, ACS Nano, 2013, 7, 3589-3597.

5 A. Walther, J. V. I. Timonen, I. Díez, A. Laukkanen and O. Ikkala, Adv. Mater., 2011, 23, 2924-2928.

6 J. Hilgert, N. Meine, R. Rinaldi and F. Schuth, Energy Environ. Sci., 2013, 6, 92-96.

7 F. Schüth, Chem. Ing. Tech., 2011, 83, 1984-1993.

8 K. Ariga, Q. Ji, M. J. McShane, Y. M. Lvov, A. Vinu and J. P. Hill, Chem. Mater., 2011, 24, 728-737.

9 Z. Weng, Y. Su, D.-W. Wang, F. Li, J. Du and H.-M. Cheng, Adv. Energy Mater., 2011, 1, 917-922.

10 H.-W. Liang, Q.-F. Guan, L.-F. Chen, Z. Zhu, W.-J. Zhang and S.-H. Yu, Angew. Chem., Int. Ed., 2012, 51, 5101-5105.

11 C. Liu, F. Li, L.-P. Ma and H.-M. Cheng, Adv. Mater., 2010, 22, E28-E62.

12 R. Li, K. Parvez, F. Hinkel, X. Feng and K. Müllen, Angew. Chem., Int. Ed., 2013, 52, 5535-5538.

13 X. Sun and Y. Li, Angew. Chem., Int. Ed., 2004, 43, 597-601. 14 X. Sun, J. Liu and Y. Li, Chem.-Eur. J., 2006, 12, 2039-2047.
15 F. Khan, D. Walsh, A. J. Patil, A. W. Perriman and S. Mann, Soft Matter, 2009, 5, 3081-3085.

16 E. M. Lambert, C. Viravaidya, M. Li and S. Mann, Angew. Chem., Int. Ed., 2010, 49, 4100-4103.

17 G. P. Mane, S. N. Talapaneni, C. Anand, S. Varghese, H. Iwai, Q. Ji, K. Ariga, T. Mori and A. Vinu, Adv. Funct. Mater., 2012, 22, 3596-3604.

18 Z.-Y. Wu, C. Li, H.-W. Liang, J.-F. Chen and S.-H. Yu, Angew. Chem., Int. Ed., 2013, 52, 2925-2929.

19 O. Ioannidou and A. Zabaniotou, Renewable Sustainable Energy Rev., 2007, 11, 1966-2005.

20 B.-H. Han, W. Zhou and A. Sayari, J. Am. Chem. Soc., 2003, 125, 3444-3445.

21 H. Wang, Q. Gao and J. Hu, J. Am. Chem. Soc., 2009, 131, 7016-7022.

22 F. Rodríguez-Reinoso, M. Molina-Sabio and M. T. González, Carbon, 1995, 33, 15-23.

23 K. Mohanty, M. Jha, B. C. Meikap and M. N. Biswas, Chem. Eng. Sci., 2005, 60, 3049-3059.

24 D.-C. Guo, J. Mi, G.-P. Hao, W. Dong, G. Xiong, W.-C. Li and A.-H. Lu, Energy Environ. Sci., 2013, 6, 652-659.

25 J. P. Paraknowitsch and A. Thomas, Macromol. Chem. Phys, 2012, 213, 1132-1145.

26 J. P. Paraknowitsch, J. Zhang, D. Su, A. Thomas and M. Antonietti, Adv. Mater., 2010, 22, 87-92.

27 D. Mecerreyes, Prog. Polym. Sci., 2011, 36, 1629-1648.

28 Y. Men, X.-H. Li, M. Antonietti and J. Yuan, Polym. Chem., 2012, 3, 871-873.

29 J. Yuan and M. Antonietti, Polymer, 2011, 52, 1469-1482.

30 J. S. Lee, H. Luo, G. A. Baker and S. Dai, Chem. Mater., 2009, 21, 4756-4758.

31 J. S. Lee, R. T. Mayes, H. Luo and S. Dai, Carbon, 2010, 48, 3364-3368.

32 J. S. Lee, X. Wang, H. Luo, G. A. Baker and S. Dai, J. Am. Chem. Soc., 2009, 131, 4596-4597.

33 J. S. Lee, X. Wang, H. Luo and S. Dai, Adv. Mater., 2010, 22, 1004-1007.

34 Q. Zhao, T.-P. Fellinger, M. Antonietti and J. Yuan, J. Mater. Chem. A, 2013, 1, 5113-5120.

35 J. Yuan, D. Mecerreyes and M. Antonietti, Prog. Polym. Sci., 2013, 38, 1009-1036.

36 Q. Zhao, T.-P. Fellinger, M. Antonietti and J. Yuan, Macromol. Rapid Commun., 2012, 33, 1149-1153.

37 Q. Zhao, M. Yin, A. P. Zhang, S. Prescher, M. Antonietti and J. Yuan, J. Am. Chem. Soc., 2013, 135, 5549-5552.

38 Q. Zhao, P. Zhang, M. Antonietti and J. Yuan, J. Am. Chem. Soc., 2012, 134, 11852-11855.

39 J. P. Paraknowitsch, A. Thomas and M. Antonietti, J. Mater. Chem., 2010, 20, 6746-6758.

40 H. Zhao, J. E. Holladay, H. Brown and Z. C. Zhang, Science, 2007, 316, 1597-1600.

41 A. Thomas, F. Goettmann and M. Antonietti, Chem. Mater., 2008, 20, 738-755. 